\title{
Parenting-related stress and psychological distress in mothers of toddlers with autism spectrum disorders
}

\author{
Annette Estes, Ph.D. ${ }^{1,2}$, Erin Olson, Ph.D. ${ }^{3}$, Katherine Sullivan, Ph.C. ${ }^{2}$, Jessica Greenson, \\ Ph.D. ${ }^{1}$, Jamie Winter, Ph.D., BCBA ${ }^{1}$, Geraldine Dawson, Ph.D ${ }^{4,5}$, and Jeffrey Munson, Ph.D. \\ ${ }^{1}$ Dept. of Speech and Hearing Sciences, University of Washington, Seattle, WA \\ ${ }^{2}$ Dept. of Psychology, University of Washington, Seattle, WA \\ ${ }^{3}$ Dept. of Education, University of Washington, Seattle WA \\ ${ }^{4}$ Dept. of Psychiatry, University of North Carolina, Chapel Hill, NC \\ ${ }^{5}$ The Autism Speaks Foundation, New York, NY \\ ${ }^{6}$ Dept. of Psychiatry, University of Washington, Seattle, WA
}

\begin{abstract}
Background-Parents of children with autism spectrum disorders (ASDs) are at risk for higher stress levels than parents of children with other developmental disabilities and typical development. Recent advances in early diagnosis have resulted in younger children being diagnosed with ASDs but factors associated with parent stress in this age group are not well understood.
\end{abstract}

\begin{abstract}
Aims-The present study examined parenting-related stress and psychological distress in mothers of toddlers with ASD, developmental delay without ASD (DD), and typical development. The impact of child problem behavior and daily living skills on parenting-stress and psychological distress were further investigated.
\end{abstract}

Methods-Participants were part of a larger research study on early ASD intervention. Parent self-report of parenting-related stress and psychological distress was utilized.

\begin{abstract}
Results-Parents of toddlers with ASD demonstrated increased parenting-related stress compared with parents of toddlers with DD and typical development. However, psychological distress did not differ significantly between the groups. Child behavior problems, but not daily living skills emerged as a significant predictor of parenting-related stress and psychological distress. This was true for both mothers of children with ASD and DD.
\end{abstract}

Conclusions-These finding suggest that parents' abilities to manage and reduce behavior problems is a critical target for interventions for young children with ASD and DD in order to improve child functioning and decrease parenting-related stress.

\section{Keywords}

Autism Spectrum Disorder; parents; distress; toddlers; parenting-stress

\section{(C) 2012 Elsevier B.V. All rights reserved.}

Publisher's Disclaimer: This is a PDF file of an unedited manuscript that has been accepted for publication. As a service to our customers we are providing this early version of the manuscript. The manuscript will undergo copyediting, typesetting, and review of the resulting proof before it is published in its final citable form. Please note that during the production process errors may be discovered which could affect the content, and all legal disclaimers that apply to the journal pertain. 
Parents of children autism spectrum disorders (ASDs) are at risk for increased levels of stress compared with parents of typically developing children and children with psychiatric and non-ASD developmental disorders (e.g., $[1,2,3,4,5])$. Parents may also be at increased risk for developing psychological symptoms, such as depression and anxiety [1,6,7]. Parents of children with ASD are faced with numerous potential challenges and daily stressors associated with parenting a child with ASD including child behavior problems [2,8,9,10] and lower levels of social reciprocity and social communication [3,11,12]. Level of child adaptive functioning and daily living skills has been investigated as a possible source of parent stress, but findings have been mixed. Three studies have reported that better adaptive functioning is associated with increased maternal well-being [10,13], while four studies report no association between parental stress and adaptive skills $[2,9,14]$. Thus, research is needed to clarify the contributors to increased stress in parents of children with ASD.

Evidence suggests that parents of younger children may experience higher levels of stress than parents of older children with ASD [12,15]. In the only longitudinal study of very young children of which we are aware, parents of toddlers (aged 18-33 months) demonstrated increased depressive symptoms with many mothers continuing to report increased symptoms two years later [16]. This suggests that parent stress and psychological distress in the toddler years may set the stage for later parent adjustment and be a critical period for parents of children with ASD. However, more research is needed to better understand how parent stress and psychological distress may manifest in parents of very young children with ASD and ultimately, how to best support parents during this period.

The toddler and preschool years bring with them a unique set of challenges and stressors that may put parents of children with ASD at risk for high levels of stress and psychological distress. The toddler years are a time when developmental difficulties are often first recognized and the complex process of early identification of ASD takes place. Parents typically report that their first concerns regarding children who go on to develop ASD emerge between 11-19 months [17,18]. Recent advances in early diagnosis have resulted in children as young as 12 months of age being diagnosed with ASDs in specialized clinics and university programs. For many families, however, there can be a long period of time between recognizing developmental challenges and obtaining a diagnosis of ASD, with an average age of diagnosis in the United States of 3-4 years depending on sample characteristics and geographical location (e.g., $[19,20,21])$. When a child first receives a diagnosis, parents must make significant personal adjustments, for example recalibrating expectations for their child's current and future functioning, and working to ameliorate the impact of ASD on personal and family life [4,22,23]. Existing evidence suggests parents of toddlers with ASD report increased stress associated with deficits in children's social relatedness, but not cognitive functioning, communication deficits, or atypical behaviors [24]. However, early diagnosis is a relatively new innovation and not yet widely available, so not enough is known about stress and adjustment among parents of very young children with ASD.

The current study investigated parenting-related stress and psychological distress in mothers of young children, ages 18-30 months. The aim was to compare parenting-related stress and psychological distress in mothers of toddlers with ASD, mothers of toddlers with developmental delay without ASD (DD), and mothers of typically developing toddlers (TYP). This study will replicate prior work by our group with a preschool age cohort (3-4 years of age; [2]). We will investigate the influence of child characteristics, including diagnostic status, problem behavior, and daily living skills, on parenting-related stress and psychological distress in these groups. We hypothesize (1) parenting-related stress and psychological distress will be higher in mothers of toddlers with ASD compared to mothers 
in the DD and TYP groups and that (2) child problem behaviors will be more strongly related to maternal parenting stress and psychological distress than child daily living skills.

\section{Methods}

\section{Participants}

Families with children between 18 and 30 months of age were recruited from the Seattle metropolitan area as part of a randomized clinical trial testing the effectiveness of the Early Start Denver Model at the University of Washington (NIH STAART). Ninety six families participated in the current study on parent stress. Participating families were assigned to one of three groups based on the diagnostic status of the toddler: autism spectrum disorder (ASD; $n=46$ ), developmentally delayed (DD; $n=25$ ), and typically developing (TYP; $n=$ 25). Diagnostic status was determined by the Autism Diagnostic Observation Schedule (ADOS; [25]), the Autism Diagnostic Interview-Revised (ADI-R; [26]), cognitive testing, and clinical best estimate diagnosis from a licensed psychologist per DSM-IV-TR diagnostic criteria. The DD group was matched to the ASD group on a measure of non-verbal mental age (mean (SD): 18.27 (4.42), 17.50 (3.17), respectively, $\mathrm{t}=.87, \mathrm{p}=.39$ ), and both DD and ASD groups were lower than the Typically developing (TYP) group (mean (SD): 22.80 $(3.45), \mathrm{F}(2,93)=18.78, \mathrm{p}<.001)$. This variable was computed from averaging ageequivalent scores on the Mullen Scales of Early Learning visual reception and fine motor scales [27]. The Mullen is a standardized measure used to assess developmental level of children from birth to 68 months. The DD group was also matched to the ASD and TYP group on chronological age (mean (SD): 22.35 (3.46), 23.50 (3.90), 22.60 (3.11) respectively, $\mathrm{F}(2,93)=0.69, \mathrm{p}=.503)$. See Table 1 .

\section{Procedure}

Participants were recruited through local parent advocacy groups, community agencies, clinics, hospital, and public schools. Upon enrollment in the study, prior to randomization to intervention, mothers completed (1) questionnaires regarding their own psychological distress and parenting stress and (2) toddler problem behavior and daily living skills. The study was approved by the University of Washington Human Subjects Review Board.

\section{Measures}

Parenting stress—The Questionnaire on Resources and Stress (QRS; [28]) is a 78-item self-report questionnaire that measures stress and burden of care in families of children with disabilities. Parents rate their agreement or disagreement on a four-point scale (strongly agree to strongly disagree) on items that tap parental feelings about their child (e.g., "I worry about what will happen to [CHILD] when I can no longer take care of him/her," "We have lost friends because of [CHILD]"). Parents provide responses on a 4-point Likert scale (strongly agree to strongly disagree) with high scores representing higher parenting-related stress. The QRS was shown to adequate reliability (internal consistency alpha from .394-. 854 ; split-half reliability $\mathrm{r}=.85$ and .89 ; stability $\mathrm{r}=.80$ ) and construct and concurrent validity. The QRS scores correlate significantly with CARS symptom-related stress scores ( $\mathrm{r}=.62$ and .67; Konstantareas et al., 1992). This study used the Parenting Stress summary score to measure parenting-related stress.

Psychological distress-The Brief Symptom Inventory (BSI; [29]) is a widely used, parent self-report that measures clinically relevant psychological symptoms. It consists of 53 items covering nine symptom dimensions: Somatization, Obsession-Compulsion, Interpersonal Sensitivity, Depression, Anxiety, Hostility, Phobic anxiety, Paranoid Ideation and Psychoticism. For the current study, the Depression and Anxiety subscales, which were highly correlated $(\mathrm{r}=0.72$ ), were averaged to create an overall measure of parent distress. 
Problem Behavior-The Aberrant Behavior Checklist (ABC; [30]) is an informant based problem behavior rating scale that consists of 58 items, each scored on a 4-point scale $(0=$ not a problem, through $3=$ problem is severe in degree). The items fall into five subscales: 1) Irritability, Agitation, Crying (15 items), 2) Lethargy, Social Withdrawal (16 items), 3) Stereotypic Behavior (7 items), 4) Hyperactivity, Non-Compliance (16 items), and 5) Inappropriate Speech (4 items). Studies have shown that the $\mathrm{ABC}$ is a reliable and valid behavior rating instrument [31].

Daily Living Skills-The Daily Living Skills domain of the Vineland Adaptive Behavior Scales (VABS; [32]) is based on a parent interview that assesses social, communication, motor, and daily living skills. The Daily Living Skills Domain includes the following subscales: Personal, Domestic, and Community. Domain and subscale scores are measured in age equivalents and standard scores. For toddlers aged two to three years the psychometric properties of the DLS scale are very good. Reliability coefficients were .91 and above for inter-rater, test-retest, and split-half reliabilities. Content, construct, and criterion-referenced validity were also established.

\section{Results}

\section{Sample demographics}

Demographic characteristics for the sample as presented in Table 1. Groups did not significantly differ in child age, gender, race, and mother's age. There was a non significant trend for the DD and TYP groups to have a higher percentage of mothers who had completed college compared to the ASD group (91\%, 92\%, and 69\% respectively). Furthermore, groups did not differ in the reported number of negative life events that had occurred in the weeks prior to enrollment in the study.

\section{Group differences in parenting stress and psychological function}

The first analysis investigated whether mothers of children with ASDs differed in terms of levels of parenting stress and psychological function (i.e. anxiety and depressive symptoms) as compared to mothers of developmentally delayed and typically developing children. Comparisons were made using orthogonal, a priori contrasts in ANOVA which contrasted the ASD and DD groups, and the ASD and TYP groups (Table 2). These planned comparisons offered a direct test of the hypothesis as well as improved power over an omnibus test and post hoc comparisons. Mothers of children with ASDs showed significantly higher parenting stress scores than the mothers of children in the DD group and the mothers of children in the TYP group. In contrast, mothers of children with ASDs showed no difference in reported levels of psychological distress, as measured by increased mean scores on the anxiety and depression scales of the BSI, compared with mothers of children in the DD and TYP groups.

\section{Group differences in child problem behavior and daily living skills}

Using the same approach, children in the ASD group demonstrated higher levels of problem behavior compared with the children in the DD and TYP groups (Table 2) as measured by the ABC Problem Behavior Composite score. Children in the ASD group also demonstrated lower Vineland Daily Living Skills scores than children in both the DD and TYP groups.

\section{Relationship between child characteristics and parenting stress and psychological distress}

Using linear regression models, we sought to understand the degree to which measurements of child problem behavior and child daily living skills account for variability in mother's 
parenting stress and psychological distress. This analysis was limited to children in the ASD and DD groups. This is because we are interested in identifying factors that may be unique to ASD, over and above factors common to the broader population of children with developmental delays. The child's diagnostic group was included in the regression model to examine whether diagnostic group membership contributed to variability in parentingrelated stress and psychological distress beyond these child variables. Table 3 shows the results in which child behavior problems, but not daily living skills or diagnostic group (ASD vs. DD) emerged as a significant predictor of parenting-related stress and psychological distress in this sample of mothers. Interaction terms were added to these models to examine whether the relationship between child behavior problems and the parental measures differed as a function of diagnostic group. No interaction terms were significant.

\section{Discussion}

In this sample of very young children (18-30 months of age), parenting-related stress was higher in mothers of toddlers with ASD than in mothers of toddlers with non-ASD developmental delays (DD) and typical development, consistent with prior research. However, psychological distress, indexed by anxiety and depressive symptoms, was not significantly different in mothers of children with ASD compared with mothers in the DD and TYP groups. This differs from prior research with mothers of preschool-aged children with ASD (e.g., [2]) and from one study of parents of children with ASD of this same age group [16]. Consistent with previous literature, child problem behavior was found to significantly contribute to parenting-related stress and psychological distress in mothers of children with ASD and DD (e.g., [9]). This held true even though children with ASD had higher levels of problem behavior than children in the DD group. Daily living skills were not related to parenting-stress or psychological distress in either the ASD or DD group. This suggests that problem behavior is a factor that increases stress in parents of all children with developmental disabilities (ASD and non-ASD) but that daily living skills are not a significant source of parenting-related stress in this age group.

The distinction between parenting-related stress and psychological distress is important to consider when evaluating these results. Parenting-related stress is directly tied to the parenting role, whereas the measure of psychological distress in this study may reflect a more stable trait. The relationship between psychological distress and rearing a child with a disability is a complex, transactional process. Thus psychological distress would be unlikely to immediately increase in response to a new situation, such as a diagnosis of ASD in a young child but may increase over time, and be more clearly evident in parents of older children. Psychological distress may not differ between mothers of ASD, DD or even typically developing children in the very early years since mothers of toddlers have not been exposed to the increased parenting demands of raising a child with ASD for as long.

Parenting-related stress was shown to be higher in mothers of children with ASD. One source of increased parenting demands demonstrated in this study and prior studies (e.g., $[2,9]$ ) was problem behavior. This suggests that child problem behavior is an important target of early autism intervention. Improving problem child behavior will make a positive impact on children with ASD themselves, but also holds the promise of improving parenting-related stress in caregivers. Toddlers with ASD in this sample, consistent with prior research, exhibited increased problem behaviors and lower daily living skills than toddlers in the DD group. However, daily living skills were not related to the increase in parenting stress. This is consistent with previous studies demonstrating that mothers of preschool children with ASD appear resilient to the additional responsibilities brought on by difficulties in child daily living skills $[2,9,14]$. This sign of resilience has important 
implications. Early intervention approaches that focus on parenting strengths may be able to build upon and reinforce the positive coping strategies parents use and increase parent resilience. If the coping skills parents bring to bear on caring for children with lower daily living skills can be generalized to other domains, perhaps parenting-stress can be reduced over the lifespan.

One limitation of this study is that a number of factors that may relate to parenting-stress and psychological distress were not measured. For example, the emotional experience of parents that are in the process of obtaining a new diagnosis of ASD was not directly explored. Most of the parents in the ASD group were receiving a first time diagnosis as part of their participation in the study. Whereas parents in the DD group were aware their child had a developmental delay prior to participation in the study. This may have been an unmeasured factor that contributed to the observed differences. Birth order could influence the amount of stress experience by parents in complicated ways. For example, having a typically developing older child may build skills and confidence with the day-to-day demands of parenting, and thus reduce the stress of parenting a child with ASD. On the other hand, this may create expectations for developmental milestones that, when not met by a child with ASD, could cause additional stress. Negative life events may also be important to consider. These types of extrafamilial factors were not measured in this study, but may increase parent stress. In addition, social support and societal factors such as a lack of acceptance of behaviors associate with autism may be important contributors to parental adjustment [33], but were outside the scope of this study.

Future studies are needed to better understand the contribution of emotional, extrafamilial, and societal factors to parenting-stress and psychological distress in families of children with ASD. Parenting demands change as children develop, so further research examining the developmental trajectory of the relationships between parenting stress and problem behavior throughout the lifespan are needed. Ultimately, it is crucial to understand changes in parenting-related stress and psychological distress over the lifespan to inform the timing of intervention delivery. These results also highlight the importance of addressing problem behaviors in interventions for young children with ASD and DD to improve child functioning and to decrease parenting-related stress. Because we know that parenting stress is increased for parents with children on the autism spectrum, the development of interventions targeting parents' abilities to manage and reduce behavior problems is a critical target for future research.

\section{Acknowledgments}

This study was supported by grant funding: ACE (NICHD) P50 HD055782 and STAART (NIH) U54 MH066399

We gratefully acknowledge the families who have participated in this study.

\section{References}

1. Bristol, MM.; Schopler, E. A developmental perspective on stress and coping in families of autistic children. In: Blacher, J., editor. Severely handicapped young children and their families. Orlando, FL: Academic Press; 1984. p. 91-141.

2. Estes A, Munson J, Dawson G, Koehler E, Zhou XH, Abbott R. Parenting stress and psychological functioning among mothers of preschool children with autism and developmental delay. Autism. 2009; 13:375-87. [PubMed: 19535467]

3. Griffith GM, Hastings RP, Nash S, Hill C. Using matched groups to explore child behavior problems and maternal well-being in children with Down syndrome and autism. J Autism Dev Disord. 2010; 40:610-19. [PubMed: 19936904] 
4. Naseef, RA. Special Children, Challenged Parents: The Struggles and Rewards of Raising a Child with a Disability. Baltimore: Paul H. Brookes; 2001.

5. Schieve LA, Blumberg SJ, Rice C, Visser SN, Boyle C. The relationship between autism and parenting stress. Pediatrics. 2007; 119:S114-S121. [PubMed: 17272578]

6. Sawyer MG, Bittman M, La Greca AM, Crettenden AD, Harchak TF, Martin J. Time demands of caring for children with autism: what are the implications for maternal mental health? J Autism Dev Disord. 2010; 40:620-28. [PubMed: 19949845]

7. Yirmiya N, Shaked M. Psychiatric disorders in parents of children with autism: A meta-analysis. J Child Psychol Psychiatry. 2005; 46:69-83. [PubMed: 15660645]

8. Gray DE, Holden WJ. Psychosocial well-being among the caregivers of children with autism. Aust N Z J Dev Disabil. 1992; 18:83-93.

9. Lecavalier L, Leone S, Wiltz J. The impact of behaviour problems on caregiver stress in young people with autism spectrum disorders. J Intellect Disabil Res. 2006; 50:172-83. [PubMed: 16430729]

10. Tomanik S, Harris G, Hawkins J. The relationship between behaviours exhibited by children with autism and maternal stress. J Intellect Dev Disabil. 2004; 29:16-26.

11. Hastings RP, Johnson E. Stress in UK families conducting intensive home-based behavioral intervention for their young child with autism. J Autism Dev Disord. 2011; 31:327-36. [PubMed: 11518485]

12. Schieve LA, Boulet SL, Kogan MD, Yeargin-Allsopp M, Boyle CA, Visser SN, et al. Parenting aggravation and autism spectrum disorders: 2007 National Survey of Children's Health. Disabil Health J. 2011; 4:143-52. [PubMed: 21723521]

13. Hall HR, Graff JC. The relationships among adaptive behaviors of children with autism, family support, parenting stress, and coping. Issues Compr Pediatr Nurs. 2011; 34:4-25. [PubMed: 21341964]

14. Beck A, Daley D, Hastings RP, Stevenson J. Mothers' expressed emotion towards children with and without intellectual disabilities. J Intellect Disabil Res. 2004; 48(Pt. 7):628-38. [PubMed: 15357682]

15. Behr, SK.; Murphy, DL. Research progress and promise: The role of perceptions in cognitive adaptation to disability. In: Turnbull, AP.; Patterson, JA.; Behr, SK.; Murphy, DL.; Marquis, JG.; Blue-Banning, MJ., editors. Cognitive coping, families, and disability. Baltimore: Brookes; 1993. p. 151-63.

16. Carter AS, Martínez-Pedraza Fde L, Gray SA. Stability and individual change in depressive symptoms among mothers raising young children with ASD: maternal and child correlates. J Clin Psychol. 2009; 65:1270-80. [PubMed: 19816954]

17. Chawarska K, Klin A, Paul R, Volkmar F. Autism spectrum disorder in the second year: Stability and change in syndrome expression. J Child Psychol Psychiatry. 2007; 48:128-38. [PubMed: 17300551]

18. DeGiacomo A, Fombonne E. Parental recognition of developmental abnormalities in autism. Eur Child Adolesc Psychiatry. 1998; 7:131-36. [PubMed: 9826299]

19. Autism and Developmental Disabilities Monitoring Network. Prevalence of autism spectrum disorders-Autism and Developmental Disabilities Monitoring Network, United States, 2006. MMWR Surveill Summ. 2009; 58:1-20.

20. Mandell DS, Morales KH, Xie M, Lawer LJ, Stahmer AC, Marcus SC. Age of diagnosis among Medicaid-enrolled children with autism, 2001-2004. Psychiatr Serv. 2010; 61:822-29. [PubMed: 20675842]

21. Shattuck PT, Durkin M, Maenner M, Newschaffer C, Mandell DS, Wiggins L, et al. Timing of identification among children with an autism spectrum disorder: Findings from a population-based surveillance study. J Am Acad Child Adolesc Psychiatry. 2009; 48:474-83. [PubMed: 19318992]

22. Altiere MJ, von Kluge S. Searching for acceptance: Challenges encountered while raising a child with autism. J Intellect Dev Disabil. 2009; 34:142-52. [PubMed: 19404835]

23. Koegel RL, Schreibman L, Loos LM, Dirlich-Wilhelm H, Dunlap G, Robbins FR, Plienis AJ. Consistent stress profiles in mothers of children with autism. J Autism Dev Disord. 1992; 22:20516. [PubMed: 1624405] 
24. Davis NO, Carter AS. Parenting stress in mothers and fathers of toddlers with autism spectrum disorders: associations with child characteristics. J Autism Dev Disord. 2008; 38:1278-91. [PubMed: 18240012]

25. Lord, C.; Rutter, ML.; DiLavore, P.; Risi, S. Autism diagnostic observation schedule. Los Angeles: Western Psychological Services; 1999.

26. Lord C, Rutter ML, LeCouteur A. Autism Diagnostic Interview-Revised: A revised version of a diagnostic interview for caregivers of individuals with possible pervasive developmental disorders. J Autism Dev Disord. 1994; 24:659-85. [PubMed: 7814313]

27. Mullen, EM. Mullen Scales of Early Learning: AGS Edition. Circle Pines, MN: American Guidance Service; 1995.

28. Konstanrtareas MM, Homatidis S, Plowright CMS. Assessing Resources and Stress in Parents of Severely Dysfunctional Children through the Clarke Modification of Holroyd's Questionnaire on Resources and Stress. J Autism Dev Disord. 1992; 22:217-34. [PubMed: 1624406]

29. Derogatis LR, Melisaratos N. The Brief Symptom Inventory: An Introductory Report. Psychol Med. 1983; 13:595-605. [PubMed: 6622612]

30. Aman, MG.; Singh, NN. Aberrant Behavior Checklist: Manual. East Aurora, NY: Slosson Educational Publications; 1986.

31. Aman, MG. Annotated bibliography on the Aberrant Behavior Checklist (ABC). Washington, DC: Author; 2002.

32. Sparrow, S.; Balla, D.; Cicchetti, D. Vineland Adaptive Behavior Scales: Interview Edition. Circle Pines, MN: American Guidance Service; 1984.

33. Sharpley C, Bitsika V, Efremidis B. Influence of gender, parental health, and perceived expertise of assistance upon stress, anxiety, and depression among parents of children with autism. J Intellect Dev Disabil. 1997; 22:19-28. 


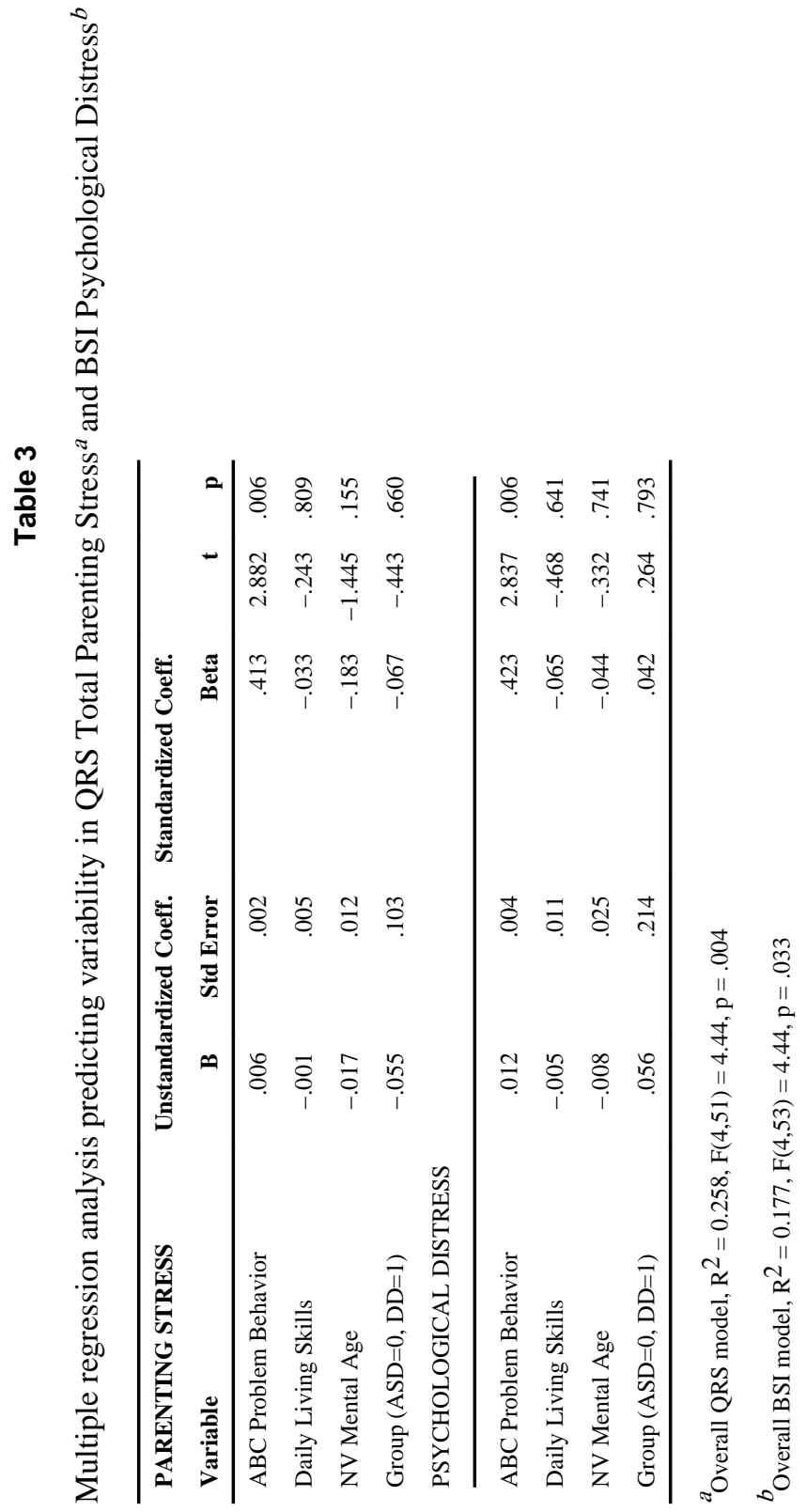

